\title{
ENGINEERING-LEVEL MODEL ATMOSPHERES FOR TITAN AND NEPTUNE
}

\author{
C.G. Justus and Aleta Duvall \\ Computer Sciences Corporation, Huntsville, AL \\ D.L. Johnson \\ NASA Marshall Space Flight Center, Marshall Space Flight Center, AL
}

\section{Introduction}

Engineering-level atmospheric models for Titan and Neptune have been developed for use in NASA's systems analysis studies of aerocapture applications in missions to the outer planets. Analogous to highly successful Global Reference Atmospheric Models for Earth (GRAM, Justus et al., 2000) and Mars (Mars-GRAM, Justus and Johnson, 2001, Justus et al., 2002) the new models are called Titan-GRAM and Neptune-GRAM. Like GRAM and Mars-GRAM, an important feature of Titan-GRAM and Neptune-GRAM is their ability to simulate quasi-random perturbations for MonteCarlo analyses in developing guidance, navigation and control algorithms, and for thermal systems design.

Figure 1 compares density-height profiles for Earth, Mars, Titan, and Neptune. Relatively low scale heights $(\sim 10 \mathrm{~km})$ make densities for Earth and Mars drop rather rapidly with altitude. Significantly higher scale height values for Titan and Neptune $(\sim 40 \mathrm{~km})$ make these atmospheres considerably "thicker". Titan's large scale height is due to its low gravity, while that for Neptune is due to its low atmospheric mean molecular weight. Vertical dotted lines in Figure 1 show density values and altitudes at which aerocapture or aerobraking maneuvers would occur on these planets.

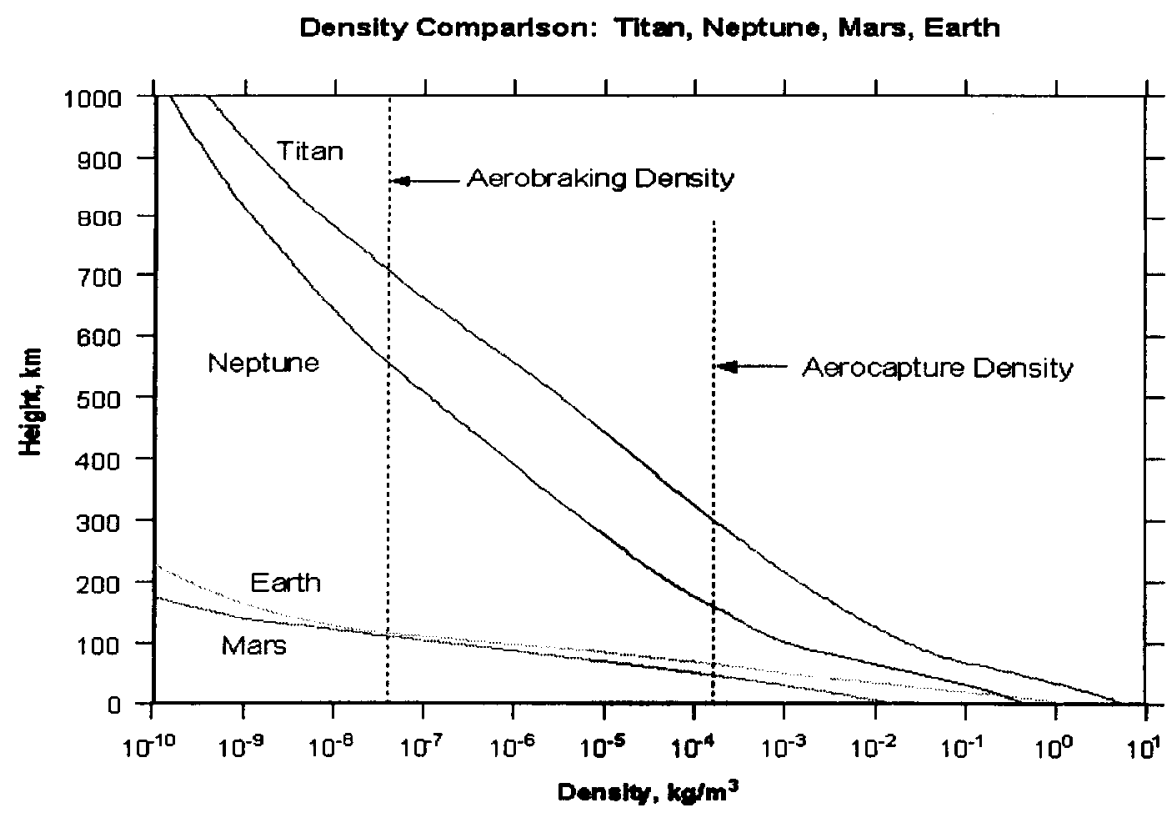

Figure 1 - Typical density versus altitude on Earth, Mars, Titan, and Neptune. 
(a)

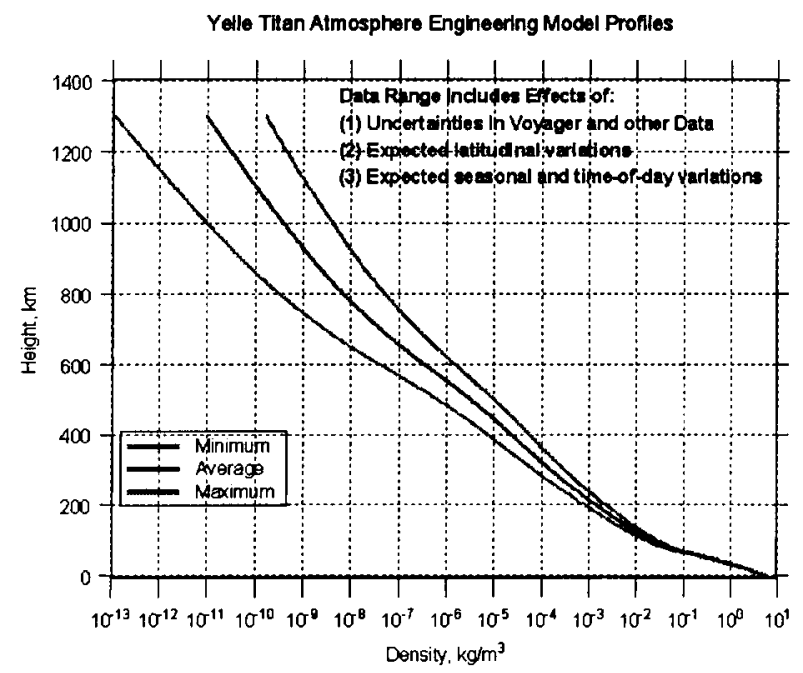

(b)

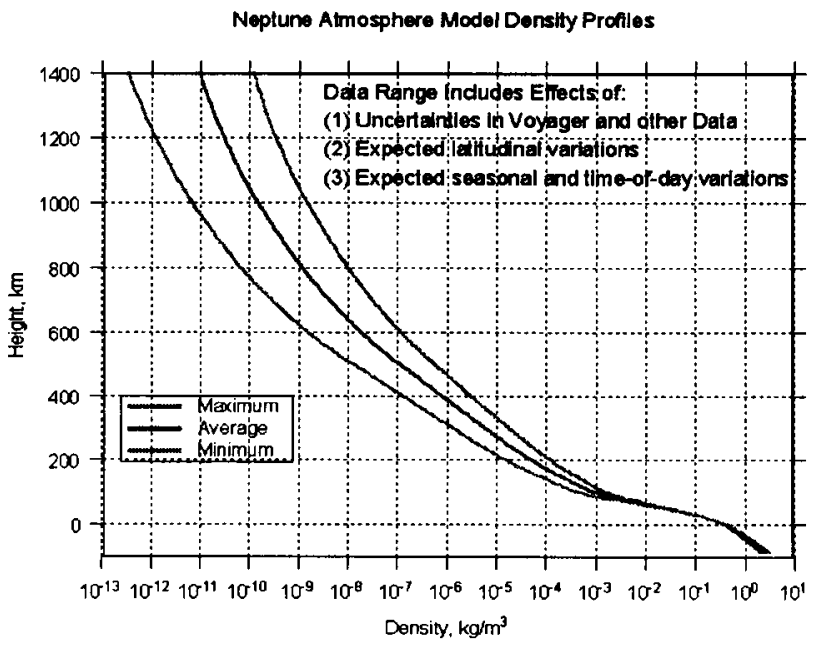

Figure 2 - Minimum, average, and maximum density versus altitude for (a) Titan (Yelle et al., 1997) and (b) Neptune (developed from data in Cruikshank, 1995).

\section{Basis for the Atmospheric Models}

In GRAM and Mars-GRAM, input values for date, time, latitude, longitude etc. are used to calculate planetary position and solar position, so that effects of latitude variation, and seasonal and time-of-day variations can be computed explicitly. A simplified approach is adopted in Titan-GRAM and Neptune-GRAM whereby these effects (as well as effects of relatively large measurement uncertainties for these planets) are represented within a prescribed envelope of minimum-averagemaximum density versus altitude. Figure 2(a) shows this envelope for Titan, for which engineering atmospheric profiles of Yelle et al. (1997) are used. For Neptune, data from Cruikshank (1995) were employed to generate a comparable envelope, shown in Figure $2 \mathrm{~b}$.

A single model input parameter (Fminmax) allows the user of Titan-GRAM or NeptuneGRAM to select where within min-max envelope a particular simulation will fall. Fminmax $=-1,0$, or 1 selects minimum, average, or maximum conditions, respectively, with intermediate values determined by interpolation (i.e. Fminmax between 0 and 1 produces values between average and maximum). Effects such as variation with latitude along a given trajectory path can be computed by user-selected representations of variation of Fminmax with latitude.

Perturbation magnitudes in Titan-GRAM and Neptune-GRAM are estimated from a methodology similar to that of Strobel and Sicardy (1997), based on expected wave saturation effects. In particular, perturbation magnitudes are modeled from an approximate fit to the wave saturation condition

$$
\rho_{\text {max }}^{\prime} / \rho_{0}=[L /(2 \pi H)]\left(1+[L /(2 \pi H)]^{2}\right)^{1 / 2}\left(d T_{0} / d z+g / C_{p}\right) /(g / R)
$$

where $H$ is pressure scale height ( $R T_{0} / g$ ), $R$ is gas constant, $g$ is acceleration of gravity, $T_{0}$ is background mean temperature, and $\mathrm{C}_{\mathrm{p}}$ is specific heat at constant pressure. Parameters $\mathrm{g} / \mathrm{Cp}$ and $\mathrm{g} / \mathrm{R}$ are known as adiabatic lapse rate and auto-convective lapse rate, respectively. Except for some slight differences in numerical factors, this is the same as equation (10) in Section 3 of the Mars-GRAM 3.34 report (Justus et al., 1996). Model perturbation magnitudes are illustrated in Figure 3. 
(a)

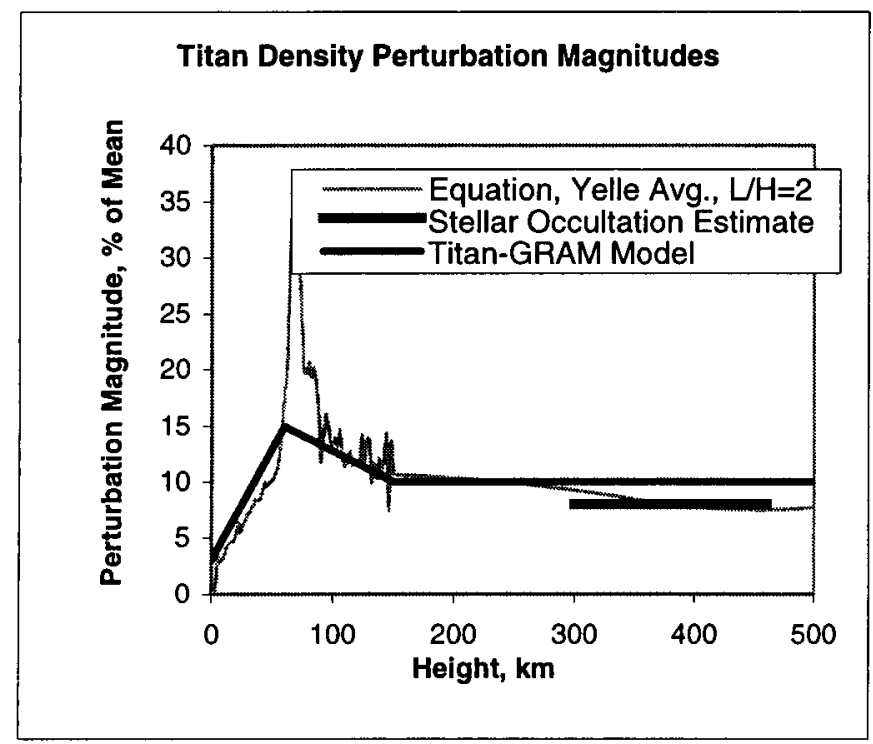

(b)

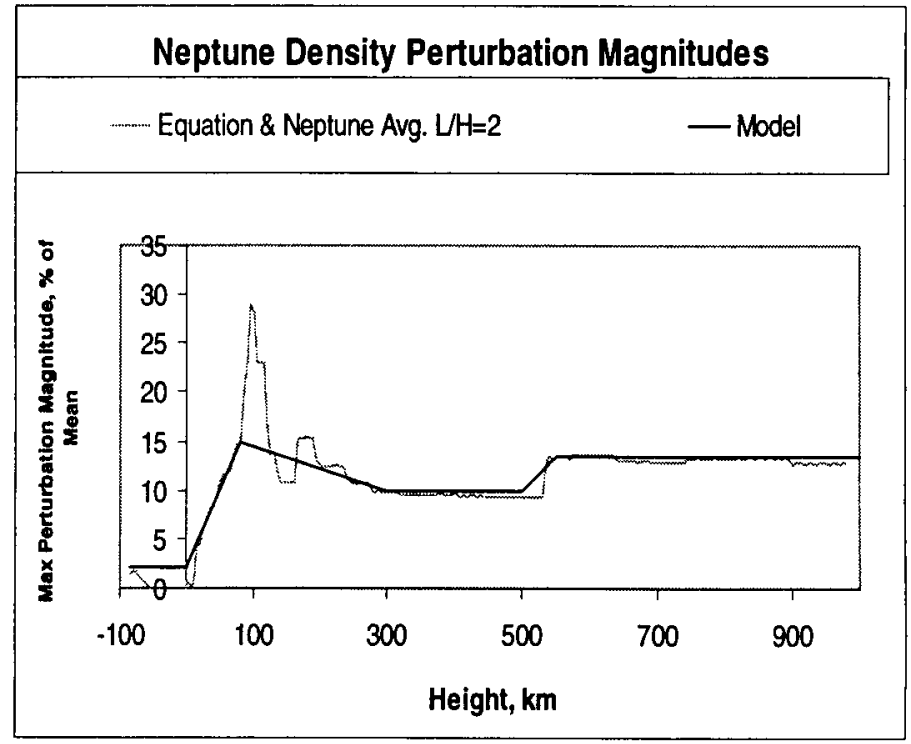

Figure 3 - Perturbation magnitudes in (a) Titan-GRAM, and (b) Neptune-GRAM. Model values approximate equation (1), with ratio $\mathrm{L} / \mathrm{H}=2$ and $\mathrm{dT} / \mathrm{dz}$ from respective average atmospheres. Textured line in (a) is estimated value from Figure 1(a) of Strobel and Sicardy (1997).

\section{Sample Model Results}

Sample Monte-Carlo outputs from Titan-GRAM and Neptune-GRAM are shown in Figure 4.

(a)

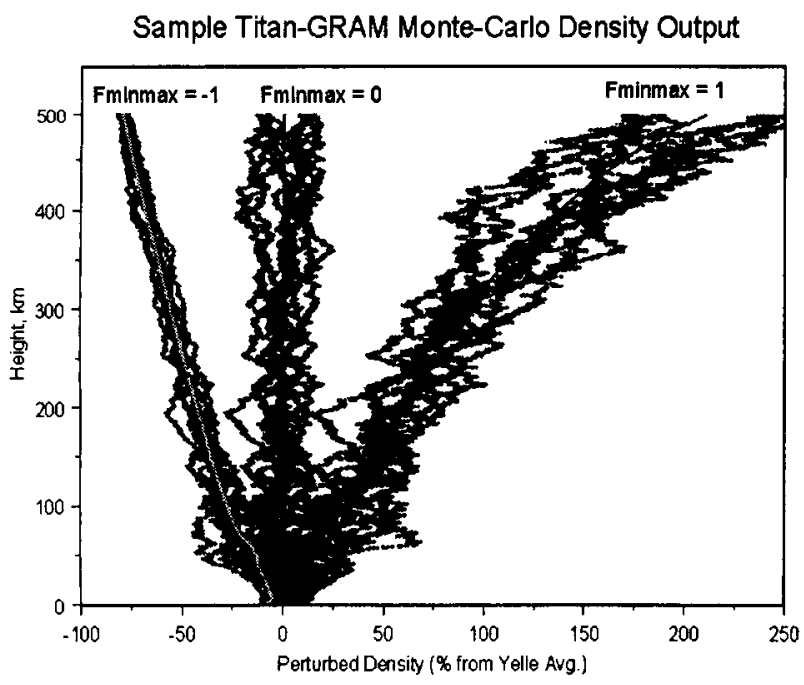

(b)

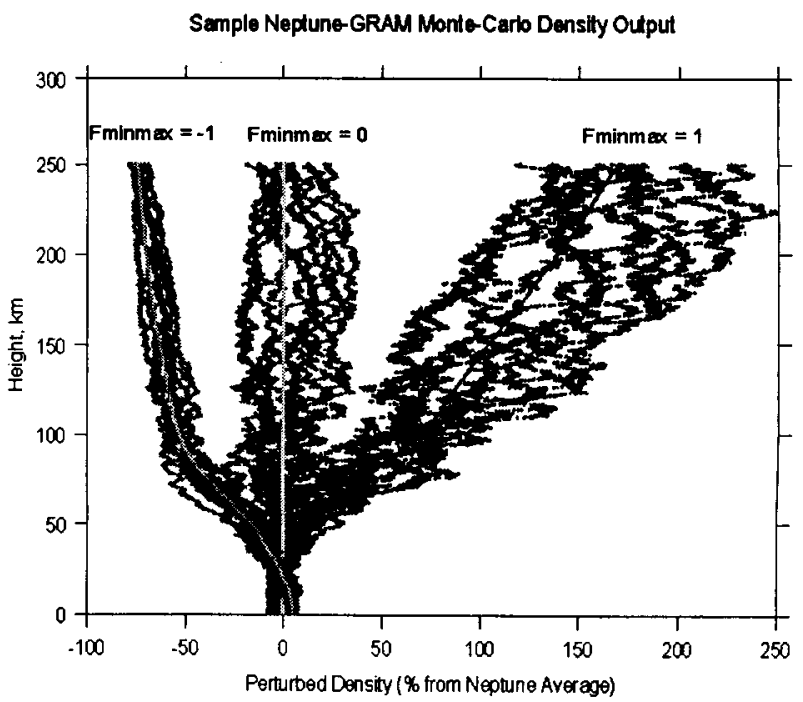

Figure 4 - Sample Monte-Carlo perturbation profiles from (a) Titan-GRAM and (b) Neptune-GRAM for Fminmax $=-1,0$ and 1. Density values are expressed as percentage deviation from average density $($ Fminmax $=0)$ profile value. 


\section{Conclusions}

Titan-GRAM and Neptune-GRAM are engineering-level atmospheric model for Titan and Neptune, suitable for a wide range of mission design, systems analysis, and operations tasks. For orbiter missions, Titan-GRAM and Neptune-GRAM applications include analysis for aerocapture or aerobraking operations, analysis of station-keeping issues for science orbits, analysis of orbital lifetimes for end-of-mission planetary protection orbits, and atmospheric entry issues for accidental break-up and burn-up scenarios. For Titan lander missions, Titan-GRAM applications include analysis for entry, descent and landing (EDL), and guidance, navigation and control analysis for precision landing. Using Titan-GRAM or Neptune-GRAM perturbation models in Monte-Carlo mode make them especially suited for design and testing of guidance, navigation, and control algorithms and for heat loads analysis of thermal protection systems.

\section{Acknowledgments}

The authors gratefully acknowledge support from the NASA/Marshall Space Flight Center InSpace Propulsion Program. Particular thanks go to Bonnie James (MSFC), Manager of the Aerocapture Technology Development Project, to Michelle M. Munk (LaRC/MSFC), Lead Systems Engineer for Aerocapture, and to Ann Trausch and Melody Herrmann (MSFC), team leads and Mary Kae Lockwood (LaRC), technical lead for the Titan/Neptune Systems Analysis study. Model user feedback and suggestions from the following are also greatly appreciated: Dick Powell, Brett Starr, and David Way (NASA LaRC), and Claude Graves and Jim Masciarelli (NASA JSC).

\section{References}

Cruikshank, D.P., editor (1995): Neptune and Triton, University of Arizona Press, 1249 pages.

Justus, C.G., B.F. James, and D.L. Johnson (1996): "Mars Global Reference Atmospheric Model (Mars-GRAM 3.34): Programmer's Guide", NASA Technical Memorandum 108509.

Justus, C. G. et al. (2000): "New Global Reference Atmospheric Model (GRAM-99) and Future Plans", invited paper C4.2-0003, COSPAR 33 ${ }^{\text {rd }}$ General Assembly, Warsaw, Poland, 16-23 July.

Justus, C. G., and D.L. Johnson (2001): "Mars Global Reference Atmospheric Model 2001 Version (Mars-GRAM 2001) Users Guide", NASA/TM-2001-210961, April.

Justus, C.G. et al. (2002): "Mars-GRAM 2000: A Mars Atmospheric Model for Engineering Applications", Advances in Space Science, 29(2), 193-202.

Strobel, D.F., and B. Sicardy (1997): Gravity Waves and Wind Shear Models", in Huygens Science, Payload and Mission, ESA SP-1177, August.

Yelle, R.V. et al. (1997): “Engineering Models for Titan's Atmosphere", in Huygens Science, Payload and Mission, ESA SP-1177, August. 